\title{
The features of modelling semiconductor lasers with a wide contact
}

\author{
Alexey Rzhanov* \\ Lomonosov Moscow State University, Physics Department, Leninskie Gory, Moscow, 119991 Russia
}

\begin{abstract}
The aspects of calculating the dynamics and statics of powerful semiconductor laser diodes radiation are investigated. It takes into account the main physical mechanisms influencing power, spectral composition, far and near field of laser radiation. It outlines a dynamic distributed model of a semiconductor laser with a wide contact and possible algorithms for its implementation.
\end{abstract}

Semiconductor laser diodes (LD) appeared in the early 60 -ies of the last century [1-2]. In 1969, due to double heterostructure implementation continuous wave (CW) LD operation at room temperature were obtained [3-4], and then the process of the LD technology development started. In the 80-ies of the last century the first works on mathematical modeling of LD dynamic and static behavior appeared [5-6].

One of the modern designs of the powerful LD with quantum well (QW) active layer and wide contact is shown in Fig. 1. Contact width w in the 8-10 W LD is about $100 \mu \mathrm{m}$.

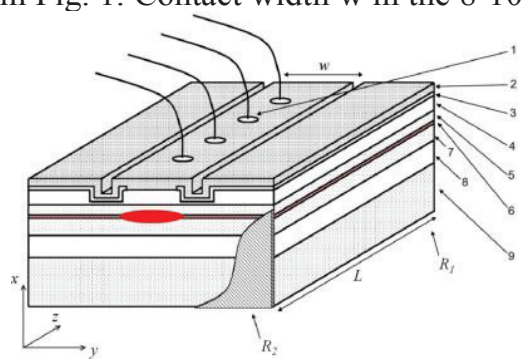

Fig. 1. Powerful injection laser: contacts (1), contact layer (2), insulating layer (3), P - emitter (4), waveguide layers (5,7), QW layer (6), N - emitter (8), substrate (9), mirrors (R1, R2) [7].

The basis of the LD models are kinetic (speed) equations that describe the balance of non-equilibrium injected carriers and photons in the active region of the laser. In their simplest form these equations write for the average values, not taking into account the spatial distribution of carriers and the optical field. In more detailed, self-consistent, models of LD the spatial interaction between the laser radiation and carriers takes into account. At other side, the waveguide permittivity profile and effective index depends on the concentration of carriers in active layer.

The number of kinetic equations in the model must match the number of lateral modes that have fallen through the generation threshold. The set of equations for carriers and

\footnotetext{
Corresponding author: rjanov@mail.ru
} 
photons concentrations along with a set of relations between the coefficients, form of the kinetic part of the model.

Second, optical part of the self-consistent model is the wave equation converted into a one-dimensional Helmholtz equation with complex variables:

$$
\frac{d^{2} \psi_{j}(\mathrm{y})}{d y^{2}}+\left(\frac{\omega_{j}^{2}}{c^{2}} \varepsilon(\mathrm{y})-\beta^{2}\right) \psi_{j}(\mathrm{y})=0
$$

where $\omega$ - optical frequency, $\beta$ - longitudinal propagation constant, $\psi$ - amplitude profiles of modes, $\varepsilon(y)$ - effective permittivity, depending on the carrier concentration, $\mathrm{c}$ - the speed of light in vacuum.

The modern LD with output power of 10-20 W have the broad pumped region ("strip") about $\mathrm{w}=100-200 \mu \mathrm{m}$ width (see Fig. 1) and the spectral band is $4-5 \mathrm{~nm}$. This is the main difficulty in creating an adequate model of LD with a broad contact.

In addition to the calculation of the transverse modes of one generation channel, for which a single set of kinetic equations and one wave equation are sufficient, in a broad cavity LD, you should consider the filamentation of radiation - decay of the optical wave at independent channels with unique light frequency and phase. As a result it is necessary to solve several equations (1) and the second kinetic equations for different channels with unknown in advance the size and frequency of the radiation. The reason for this phenomenon is the low coherence length of LD radiation (about 1-5 sm) which prohibits the phase locking between channels. Calculations and experiment show that at a cavity length of 1000 microns, and the width of the active region of the LD resonator of $100 \mu \mathrm{m}$ not less than 3 channels is shaped [8-9]. The solution of this problem, associated with forced separation of the channels, does not lead to adequate results of calculations, although for some cases allows to qualitatively describing the dynamic pattern of switching between the channels and transverse modes [9]. The model as a self-consistent set of equations grows several times in this approach, as the number of equations (1) in the model must match the number of channels. The main question is determination of spatial dimensions of each channel, as well as the energy exchange between non phase-locked areas of generation. This task until now has not yet resolved.

A separate topic for consideration is the accounting processes associated with heating of the active region of LD. This requires the solution of the heat equation in parallel with the dynamic task. Such calculations in the form of full self-consistent problem yet due to the fact that it requires huge computer capacities. Thus, the separate problems of dynamics and thermal conductivity gives quite adequate results.

There are several physical mechanisms that influence the dynamics of LD radiation, for example, "spectral hole burning", the polarization effects due to light and heavy holes in QW stressed layers, and so on. However, to include in the model many mechanisms does not work due to the occurrence of instabilities and because of computer restrictions on the amount of computation.

\section{References}

[1] Z.I. Alferov, R.F. Kazarinov. Copyright № 181737. Patent № 95084030.03 .1963$.

[2] H. Kroemer, Proc. IEEE 51, 12 (1963)

[3] Z.I. Alferov et al., Sov. Phys. Tech. Semicond. 3, 1328 (1969)

[4] I. Hayashi et al., Appl. Phys. Lett. 17, 2 (1970)

[5] J. Buus, IEEE J. Quant. Electron. QE-18, 7 (1982)

[6] J. Buus, IEEE J. Quant. Electron. QE-19, 6 (1983)

[7] I.S. Tarasov, Quant. Electron. 40, 8 (2010)

[8] O.I. Koval, A.G. Rzhanov, G.A. Solovyev, Phys. of WP, 21(4), 287 (2013)

[9] V.V. Bliznyuk, M.A. Brit, I.S. Gadaev et al., Bull. RAS: Physics 79, 12 (2015) 